\title{
A New Approach to Communications Using Chaotic Signals
}

\author{
Ned J. Corron, Member, IEEE, and Daniel W. Hahs
}

\begin{abstract}
In this paper, a new approach for communication using chaotic signals is presented. In this approach, the transmitter contains a chaotic oscillator with a parameter that is modulated by an information signal. The receiver consists of a synchronous chaotic subsystem augmented with a nonlinear filter for recovering the information signal. The general architecture is demonstrated for Lorenz and Rossler systems using numerical simulations. An electronic circuit implementation using Chua's circuit is also reported, which demonstrates the practicality of the approach.
\end{abstract}

Index Terms - Chaos, communications with chaos, synchronization.

\section{INTRODUCTION}

$\mathbf{I}$ $\mathrm{N}$ THIS PAPER, we present a new, general design ${ }^{1}$ for constructing viable communication systems using chaotic waveforms. This approach uses parameter modulation of chaotic oscillators for transmitting an information signal and a synchronous subsystem augmented with a nonlinear filter for detecting and recovering the transmitted information. Specifically, we claim that our construction of a nonlinear filter is a new development that may offer improved performance for practical chaotic communication systems.

A recent, growing body of literature supports the possibility of practical communication using chaotic signals. In particular, recent advances in the understanding of nonlinear circuits have shown that chaotic oscillators can synchronize or become entrained [1]-[4]. This surprising observation appears to contradict the very essence of chaos: complex, unpredictable dynamics of a deterministic system characterized most commonly as extreme sensitivity to initial conditions [5]. However, if certain requirements are met, then a chaotic circuit (called the driving system) can be designed to drive a similar system (the receiving circuit or subsystem) and obtain a correlated response. Despite the recent discovery of chaotic synchronization and its use for communications, a vast amount of research in this area has been presented in the literature. Pecora has assembled an extensive list of references [6]; in addition, recent conference proceedings contain and reference additional important papers [7]-[11].

As reported in the literature, synchronization of chaotic systems suggests the possibility for communication using chaotic waveforms as carriers, perhaps with application to

Manuscript received December 14, 1995; revised June 25, 1996. This paper was recommended by Associate Editor T. Endo.

The authors are with Dynetics, Inc., Huntsville, AL 35814 USA.

Publisher Item Identifier S 1057-7122(97)02075-8.

${ }^{1}$ Appropriate filings have been made with the U. S. Patent Office. secure communication. The obvious approach uses a chaotic oscillator as the transmitter and a synchronous chaotic system for the receiver, and several designs have been suggested that fit within this construct [8]. The variation in these designs lies in the methods for injecting an information signal at the transmitter and recovering it at the receiver.

One approach employed to achieve secure communications uses a chaotic signal to mask the sensitive information signal. In this approach, a synchronous chaotic system is used in the receiver to identify the chaotic part of the signal, which then is subtracted to reveal the information signal. Difficulties in this approach have been highlighted in the literature [12]; however, several researchers have successfully demonstrated this approach in simulation and with hardware [13]-[15]. Short investigated the level of security afforded by this approach, concluding that chaotic masking can offer some privacy but is not yet capable of providing a high level of communication security [16].

Several similar methods for transmission of digital signals have been proposed. Oppenheim et al. proposed a chaotic switching method in which information is transmitted by switching the transmitted signal between two chaotic sources [13]. The resulting waveform is a sequence of chaotic bursts, each generated by an oscillator corresponding to the value of the binary information signal. Originally, their approach used a likelihood ratio test to identify the dynamics of each burst, but subsequent research used a replica of the drive signal obtained using a synchronized chaotic system to detect the presence or absence of synchronization [15]. Parlitz et al. demonstrated a similar approach using Chua's circuit [17]. Dedieu et al. demonstrated a binary version of a more elaborate shift-key approach in which an information signal is encoded using a different chaotic attractor for each symbol in the message [12]. Upon reception, separate receiver subsystems, one for each possible symbol, are used to identify the bursts by detecting which of the multiple receiver subsystems has synchronized. This approach is limited to digital modulation and requires complex detection logic in the receiver. Recently, Celka has analyzed a similar method that can be implemented using time-delayed feedback optical systems [18].

A related approach for analog communication is achieved by detecting parameter mismatch between the transmitter and the receiver. The mismatch, which is intentionally introduced at the transmitter, is detected by comparing the received signal with a replica generated using cascaded synchronous subsystems [19]. This scheme recognizes that the magnitude of the difference in these signals is proportional to the parameter 
mismatch, thereby providing a crude method for demodulation. However, this approach suffers when noise is present in the communication channel.

Several researchers have utilized an inverse system approach for communication using chaos. In this approach, an information signal is mixed with a chaotic waveform using a functional coding operation. The receiver consists of a synchronous chaotic oscillator, from which the information signal is recovered by effectively inverting the coding operation used in the transmitter. A sequence of papers documents the development of the inverse system approach [20]-[23]. A recent paper by Feldmann $e t$ al. generalizes the inverse system approach [24]. Recently, Dmitriev et al. reported successful experiments using speech and music signals in this type of a system [25].

In addition to these approaches based on continuous time systems, several approaches for communication with chaos in discrete time systems have been advanced [26], [27]. Sophisticated binary communications systems have also been presented by Hayes et al. [28] and by Bernhardt [29].

In contrast to these previous approaches, we propose a communications system in which a novel nonlinear filter is used to recover the information signal. In the transmitter, an analog information signal is injected through modulation of a parameter in the drive system, thereby directly influencing the chaotic waveform in a complex manner. This differs from chaotic masking approaches, which merely combine the information and chaos signals. At the receiver, the signal is recovered using a dynamic filter. Although this approach fits within the most general definition of an inverse system, such as given in [8], our approach differs significantly from other inverse systems reported in [20]-[25], which all use an instantaneous, unfiltered inversion of the coding function. As a result, the receiver in our approach is more tolerant to channel effects such as noise and distortion. The proper choice of drive channel and modulation parameter maintains synchronization in the receiver, independent of the modulation. Further, the system architecture we present is general and provides a design procedure that can be applied to a wide array of nonlinear oscillators. Resulting systems often can be easily and inexpensively implemented using simple analog circuitry, making this approach feasible for practical communications applications.

We now outline the remainder of the paper. In Section II, we present a theoretical development for the communications system including a description of the nonlinear filter. In Section III, we present simulation results that demonstrate communications using both Lorenz and Rossler systems. In Section IV, we describe an electrical implementation using Chua's circuit by which we have successfully demonstrated communications using a chaotic carrier. Finally, in Section V, we conclude with some comments regarding applications and the status of our current research and development efforts.

\section{General THEORY}

The general format of our approach is shown in Fig. 1. In the transmitter, an analog information signal is encoded on

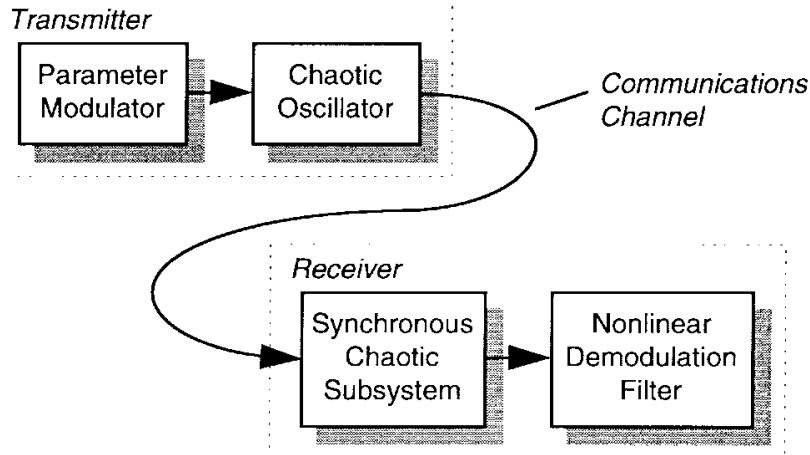

Fig. 1. Block diagram of the communications architecture incorporating parameter modulation of a chaotic oscillator and a nonlinear demodulation filter.

the carrier using modulation of a parameter in the chaotic oscillator. In the receiver, a synchronous chaotic subsystem is augmented with a filter designed specifically to continuously extract the signal from the modulated waveform. Proper choice of drive channel and modulation parameter assures synchronization in the receiver, independent of the modulation.

The general theory underlying our approach is best described mathematically. For definiteness, the theory is presented for a third-order oscillator, although it is easily generalized to systems of any order. Consider an oscillator of the form

$$
\begin{aligned}
& \dot{x}=f(x, y, z ; \lambda) \\
& \dot{y}=g(x, y, z) \\
& \dot{z}=h(x, y, z)
\end{aligned}
$$

where $x, y$, and $z$ define the states of the system, $\lambda$ is a parameter in the system, and the independent variable is time $t$. The oscillator in (1) may or may not be chaotic; however, it is expedient to assume that the oscillator is chaotic and that the system behavior changes smoothly with $\lambda$ over some continuous region of parameter values. The system (1) comprises the transmitter in Fig. 1.

A synchronous subsystem for (1) can be constructed of the form

$$
\begin{aligned}
& \dot{y}_{r}=g\left(x, y_{r}, z_{r}\right) \\
& \dot{z}_{r}=h\left(x, y_{r}, z_{r}\right)
\end{aligned}
$$

where the $x$-state from the drive system (1) is transmitted to the receiving system (2). Pecora and Carroll have shown that, quite remarkably, the states $y_{r}$ and $z_{r}$ in (2) can approach the original states $y$ and $z$ in (1); when they do, it is said that the receiving subsystem (2) synchronizes with the nonlinear drive system in (1) [1]. Further, they have determined necessary conditions for synchronization based on conditional Lyapunov exponents. The subsystem (2) is contained in the receiver as shown in Fig. 1.

The drive (1) and synchronous receiver subsystem (2) exhibit a special form with regard to the parameter $\lambda$ : subsystem (2) is purposely chosen to be independent of the parameter $\lambda$. This special form is an essential element of our approach, in which the parameter $\lambda$ is ideally suited for modulating the 

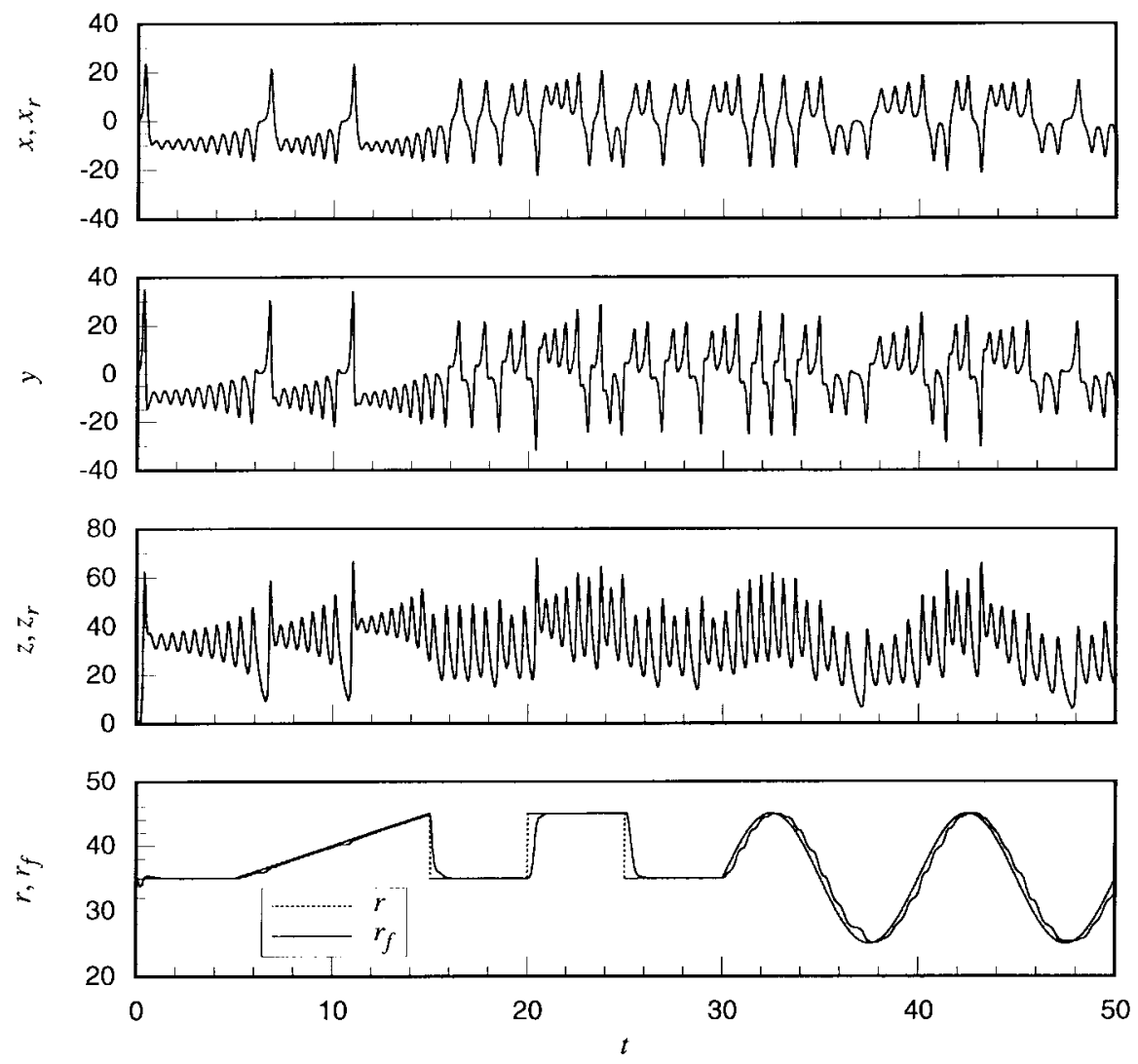

Fig. 2. Simulation results for a communication system based on a Lorenz chaotic oscillator.

chaotic waveform that is transmitted from (1) to (2). Hence, we let

$$
\lambda=\lambda(t)
$$

be a prescribed function of time that represents the information to be communicated. In (3), we assume that $\lambda$ is well behaved so as to not destroy the underlying structure of the system in (1). The quantity in (3) comprises the input parameter modulation signal shown in Fig. 1.

The proposition that $\lambda$ is ideal for parameter modulation is supported by two observations. First, since the subsystem (2) is independent of $\lambda$, the receiver can be "perfectly tuned" to the transmitter in (1) regardless of the modulation. In contrast, if $\lambda$ were present in the receiver system (2), a parameter mismatch would be unavoidable as the signal is modulated. This mismatch would compromise the ability of the receiver to synchronize with the transmitter, and the capability for communication could be reduced. Second, the $x$-equation in (1), which is not present in subsystem (2), can be utilized within the receiver to demodulate the signal.

As a first consideration, demodulation can be performed as

$$
\lambda=f^{-1}\left(x, y_{r}, z_{r} ; \dot{x}\right)
$$

where $f^{-1}$ represents the inverse function obtained by solving the $x$-equation for the parameter $\lambda$. In principle, everything on the right-hand side of (4) is known and $\lambda$ can be estimated; in practice, this approach suffers for two reasons. First, $\dot{x}$ must be estimated from $x$ at the receiver, and differentiation magnifies errors due to noise in the system. Second, the inverse function in (4) may contain singularities that adversely impact parameter estimates.

The key innovation in our communications system is a nonlinear filter that demodulates the signal in (3) and avoids the two problems indicated in the previous paragraph. To present this filter, the system (1) is restricted by assuming $f$ is linear in $\lambda$. That is,

$$
f(x, y, z ; \lambda)=f_{0}(x, y, z)+\lambda f_{1}(x, y, z) .
$$

Often, the form in (5) can be obtained if $f$ is nonlinear by assuming small variations of $\lambda$. With this assumption, the naive estimate in (4) is simply

$$
\lambda=\frac{\dot{x}-f_{0}\left(x, y_{r}, z_{r}\right)}{f_{1}\left(x, y_{r}, z_{r}\right)} .
$$

In (6), singularities are encountered whenever $f_{1}=0$.

The process of designing a robust filter for demodulation begins by utilizing the $x$ equation in (1), which is not present in the synchronous subsystem (2). With the restriction in (5), the first equation of (1) is

$$
\dot{x}=f_{0}\left(x, y_{r}, z_{r}\right)+\lambda f_{1}\left(x, y_{r}, z_{r}\right) .
$$

It is desirable to integrate (7) to get rid of $\dot{x}$; however, it is undesirable to introduce a requirement to know something about the system's initial conditions. To this end, adding a decay term $k x$ to both sides of (7) and multiplying by an integration factor $e^{k t}$ yields

$$
\frac{d}{d t}\left(x e^{k t}\right)=e^{k t}\left[f_{0}\left(x, y_{r}, z_{r}\right)+\lambda f_{1}\left(x, y_{r}, z_{r}\right)+k x\right]
$$



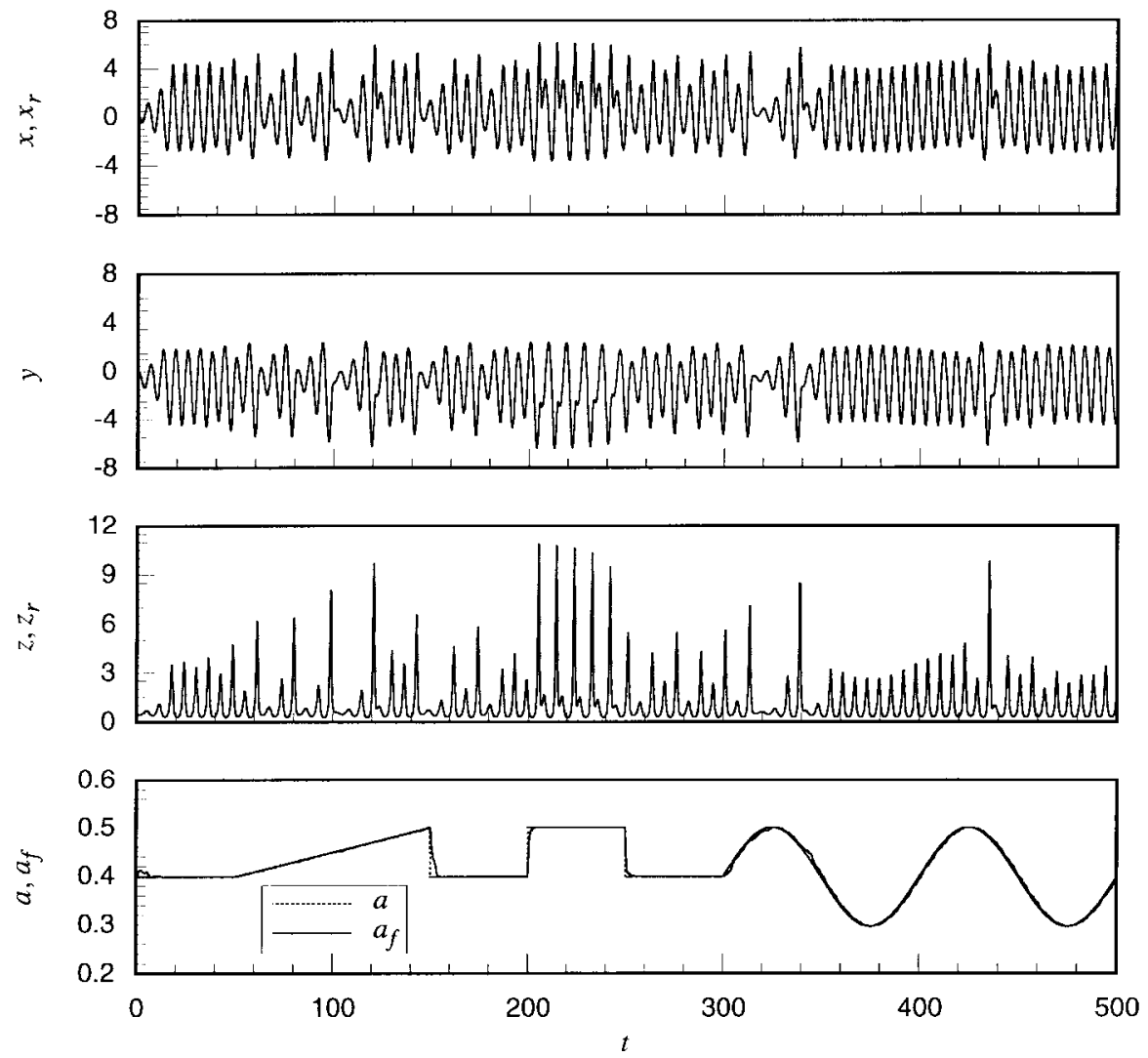

Fig. 3. Simulation results for a communication system based on a Rossler chaotic oscillator.

In this, $k>0$ is an arbitrary constant, which can be adjusted to improve the output of the demodulation filter. Integrating from a start time $t_{0}$ to the current time $t$ and dividing by the integration factor gives

$$
\begin{aligned}
x-x_{0} e^{k\left(t_{0}-t\right)}= & \int_{t_{0}}^{t}\left[f_{0}\left(x, y_{r}, z_{r}\right)+k x\right] e^{k(\tau-t)} d \tau \\
& +\int_{t_{0}}^{t} \lambda f_{1}\left(x, y_{r}, z_{r}\right) e^{k(\tau-t)} d \tau
\end{aligned}
$$

where $x_{0}$ is an initial condition applied at $t=t_{0}$. Rigorously, $\lambda$ varies with time; however, it is assumed that $\lambda$ varies slowly compared to the time constant contained in $k$. Thus, $\lambda$ can be factored out of the integral in (9). In addition, the term in (9) containing the initial condition $x 0$ becomes small as time increases; therefore, it is negligible. As a result, (9) can be simplified to provide an estimate for the modulation parameter as

$$
\hat{\lambda}=\frac{x-w_{0}}{w_{1}}
$$

where

$$
w_{0}=\int_{t_{0}}^{t}\left[f_{0}\left(x, y_{r}, z_{r}\right)+k x\right] e^{k(\tau-t)} d \tau
$$

and

$$
w_{1}=\int_{t_{0}}^{t} f_{1}\left(x, y_{r}, z_{r}\right) e^{k(\tau-t)} d \tau .
$$

The estimate given by (10), (11), and (12) is an improvement over the estimate in (6) in that $\dot{x}$ is not required.
The quantities $w_{0}$ and $w_{1}$ are not easily computed using the explicit forms in (11) and (12). Differentiating (11) and (12) using Leibnitz' rule gives the simpler forms

$$
\begin{aligned}
& \dot{w}_{0}=f_{0}\left(x, y_{r}, z_{r}\right)+k x-k w_{0} \\
& \dot{w}_{1}=f_{1}\left(x, y_{r}, z_{r}\right)-k w_{1}
\end{aligned}
$$

with initial conditions $w_{0}\left(t_{0}\right)=0$ and $w_{1}\left(t_{0}\right)=0$. The system in (13) is more practical for continuous integration than (11) and (12). The filter equations in (13) are contained in the receiver shown in Fig. 1.

The instantaneous demodulation estimate in (10) is singular for $w_{1}=0$, and an estimate for will suffer for small $w_{1}$. In practice, this singularity appears as "spikes" in the estimate for $\hat{\lambda}$. To remove these "spikes," a low-pass filter is defined as

$$
\dot{\lambda}_{f}=q_{f}\left(\hat{\lambda}-\lambda_{f}\right)
$$

where $\hat{\lambda}$ is given by (10). To avoid the singularity, the filter parameter $q_{f}$ is defined as

$$
q_{f}=\frac{q\left|w_{1}\right|}{1+\left|w_{1}\right|}
$$

where $q$ is an arbitrary parameter that sets the time constant of the filter. Combining (10), (14), and (15) yields

$$
\dot{\lambda}_{f}=\frac{q \operatorname{sgn}\left(w_{1}\right)}{1+\left|w_{1}\right|}\left(x-w_{0}-w_{1} \lambda_{f}\right)
$$

where sgn represents the signum function. The quantity $\lambda_{f}$ is then a filtered estimate for the modulation parameter $\lambda$ and 
comprises the recovered modulation signal shown in Fig. 1. The filter equation (16) is the final element of the receiver shown in Fig. 1.

In summary, the system in (1) constitutes the transmitter, with a modulation prescribed through $\lambda(t)$. The synchronous subsystem in (2) constitutes the first stage of a receiver for the chaotic waveform $x(t)$. The system in (13) and (16) is a filter designed to demodulate the signal, thereby recovering the information encoded using the modulation parameter $\lambda$.

\section{COMPUTER Simulations}

In this section, simulation results are presented for communication systems built using Lorenz and Rossler oscillators. Together with the hardware implementation using Chua's circuit presented in Section IV, these demonstrations show the wide applicability of our general approach.

The Lorenz system has been widely used for studying chaos and synchronization [5]. This third-order system is

$$
\begin{aligned}
& \dot{x}=\sigma(y-x) \\
& \dot{y}=r x-y-x z \\
& \dot{z}=x y-b z
\end{aligned}
$$

where $x, y$, and $z$ are the states of the system and $\sigma, r$, and $b$ are fixed parameters.

It has been shown that two stable subsystems can be constructed from (17): they are the $x-z$ and $y-z$ subsystems [1]. For the present example, the $x-z$ subsystem is used to demonstrate the communication system. Thus, $y$ is the transmitted component of (17); correspondingly, $r=r(t)$ is chosen for the modulation parameter.

Explicitly, the system in (17) constitutes the transmitter system. The receiver subsystem is

$$
\begin{aligned}
& \dot{x}_{r}=\sigma\left(y-x_{r}\right) \\
& \dot{z}_{r}=x_{r} y-b z_{r} .
\end{aligned}
$$

The nonlinear filter for demodulating the signal is

$$
\begin{aligned}
& \dot{w}_{0}=(k-1) y-x_{r} z_{r}-k w_{0} \\
& \dot{w}_{1}=x_{r}-k w_{1} \\
& \dot{r}_{f}=\frac{q \operatorname{sgn}\left(w_{1}\right)}{1+\left|w_{1}\right|}\left(y-w_{0}-w_{1} r_{f}\right)
\end{aligned}
$$

where $k$ and $q$ are filter parameters that are chosen for optimal performance. In (19), $r_{f}$ is the filtered estimate of the transmitted signal encoded using $r=r(t)$ in (17).

An example of communication with the Lorenz system is shown in Fig. 2. This example was generated by integrating (17), (18), and (19) numerically. For this example, $b=$ $8 / 3, \sigma=10, k=20$, and $q=20$. The first three plots show the $x, y$, and $z$ states derived from (17). The first and third plots have the $x_{r}$ and $z_{r}$ states from (18) overlaid; however, synchronization is achieved very quickly on the shown time scale, and the corresponding states in the transmitter and receiver are indistinguishable in the plots. The fourth plot shows the applied modulation $r$ and the recovered signal $r_{f}$ derived via (19). For the range of $r$ used, the oscillator remains chaotic. The agreement in the fourth plot demonstrates the capability of the proposed approach to transmit and receive information using a chaotic carrier.

The Rossler system is also widely used for studying the nonlinear dynamics of chaos [5]. This system is

$$
\begin{aligned}
& \dot{x}=-y-z \\
& \dot{y}=x+a y \\
& \dot{z}=b+z(x-c)
\end{aligned}
$$

where $x, y$, and $z$ are the states of the system and $a, b$, and $c$ are fixed parameters.

For synchronization, only the $x-z$ subsystem is stable, and then only for restricted parameter ranges [3]. Thus, this subsystem is used to demonstrate communication, with $y$ as the transmitted signal and $a=a(t)$ the corresponding modulation parameter.

The receiver subsystem is

$$
\begin{aligned}
& \dot{x}_{r}=-y-z_{r} \\
& \dot{z}_{r}=b+z_{r}\left(x_{r}-c\right)
\end{aligned}
$$

and the demodulation filter is

$$
\begin{aligned}
& \dot{w}_{0}=x_{r}+k y-k w_{0} \\
& \dot{w}_{1}=y-k w_{1} \\
& \dot{a}_{f}=\frac{q \operatorname{sgn}\left(w_{1}\right)}{1+\left|w_{1}\right|}\left(y-w_{0}-w_{1} a_{f}\right) .
\end{aligned}
$$

An example of modulation and demodulation for the Rossler system is shown in Fig. 3. For this example, (20)-(22) were integrated numerically using $b=2, c=4, k=8$, and $q=8$. Again, it is noted that the filter demodulates the chaotic waveform and extracts the encoded signal.

\section{HARDWARE DEMONSTRATION}

An example of our communication system has been implemented using an electrical circuit. This implementation is based on Chua's circuit, which is a simple electronic circuit that is widely used for demonstrating nonlinear dynamics and chaos [30].

The transmitter, shown in Fig. 4, is described mathematically by a dimensionless system of ordinary differential equations, which are

$$
\begin{aligned}
& \frac{d x}{d \tau}=\alpha[y-(1+\gamma) x-\phi(x)+\gamma \lambda] \\
& \frac{d y}{d \tau}=x-y+z \\
& \frac{d z}{d \tau}=-\beta y
\end{aligned}
$$




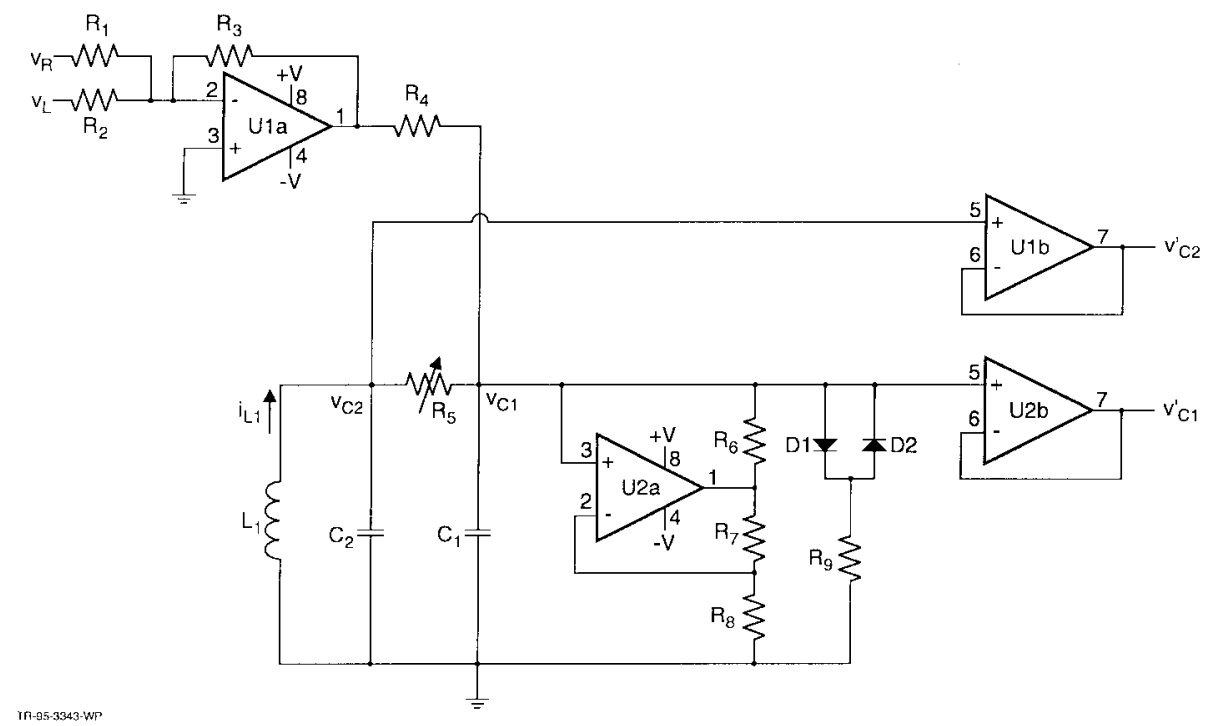

Fig. 4. Schematic for an electrical circuit realization of a modulated chaotic transmitter incorporating Chua's circuit.

where

$$
\phi(x)=a x+\frac{b-a}{2}(|x+1|-|x-1|) .
$$

In the system (23), the nondimensional independent variable $\tau$ is related to time $t$ as

$$
\tau=\frac{t}{R_{5} C_{2}}
$$

The dependent states are

$$
\begin{aligned}
& x=\frac{v_{C 1}}{V_{\text {on }}} \\
& y=\frac{v_{C 2}}{V_{\text {on }}} \\
& z=\frac{R_{5} i_{L 1}}{V_{\text {on }}}
\end{aligned}
$$

where $V_{\text {on }}$ is the voltage drop for a diode in the forward bias ( $\sim 0.7 \mathrm{~V}$ for a silicon diode). The various dimensionless parameters are defined as

$$
\begin{aligned}
\alpha & =\frac{C_{2}}{C_{1}} \\
\beta & =\frac{R_{5}^{2} C_{2}}{L_{1}} \\
\gamma & =\frac{R_{5}}{R_{4}} \\
a & =\frac{R_{5}}{R_{2}}-\frac{R_{5} R_{7}}{R_{6} R_{8}} \\
b & =-\frac{R_{5} R_{7}}{R_{6} R_{8}} .
\end{aligned}
$$

The input modulation is represented as

$$
\lambda=\frac{R_{3}}{V_{\text {on }}}\left(\frac{v_{R}}{R_{1}}+\frac{v_{L}}{R_{2}}\right)
$$

where $v_{R}$ and $v_{L}$ are two input voltages. The use of two separate inputs is motivated by the stereo channels available in common audio sources (e.g., a portable cassette player) used for demonstrating the communication system. Since this demonstration system can transmit only one information channel, a simple mixer is incorporated at the input to generate a monophonic representation of the audio signal.

The system in (23) reverts to the standard, unmodulated Chua system for $\gamma=0$. In the circuit of Fig. 4, this is easily obtained by removing $R_{4}$.

The receiver, shown in Fig. 5, is modeled nondimensionally as

$$
\begin{aligned}
\frac{d y_{r}}{d \tau} & =x-y_{r}+z_{r} \\
\frac{d z_{r}}{d \tau} & =-\beta y_{r} \\
\frac{d w_{0}}{d \tau} & =\alpha\left[y_{r}-(1+\gamma) x-\phi(x)\right]+k x-k w_{0} \\
\frac{d \lambda_{f}}{d \tau} & =q_{f}\left[\frac{w_{0}-x}{\gamma}-\lambda_{f}\right] .
\end{aligned}
$$

Due to the form of the modulation, the equation for $w_{1}$ (as presented in (13) of the general theory) can be solved analytically, yielding $w_{1} \sim-(1 / \gamma)$ as $\tau \rightarrow \infty$. Thus, this equation is not required in (29), and the simpler form (14) can be used instead of (16) for the last equation in (29).

In the dimensionless system (29), the dependent states are

$$
\begin{aligned}
y_{r} & =\frac{v_{C 3}}{V_{\text {on }}} \\
z_{r} & =\frac{R_{5} i_{L 2}}{V_{\text {on }}} \\
w_{0} & =\frac{v_{C 4}}{V_{\text {on }}} \\
\lambda_{f} & =\frac{v_{C 5}}{\gamma V_{\text {on }}} .
\end{aligned}
$$

The two filter constants are

$$
\begin{aligned}
k & =\alpha \\
q_{f} & =\frac{R_{5} C_{2}}{R_{24} C_{5}}
\end{aligned}
$$




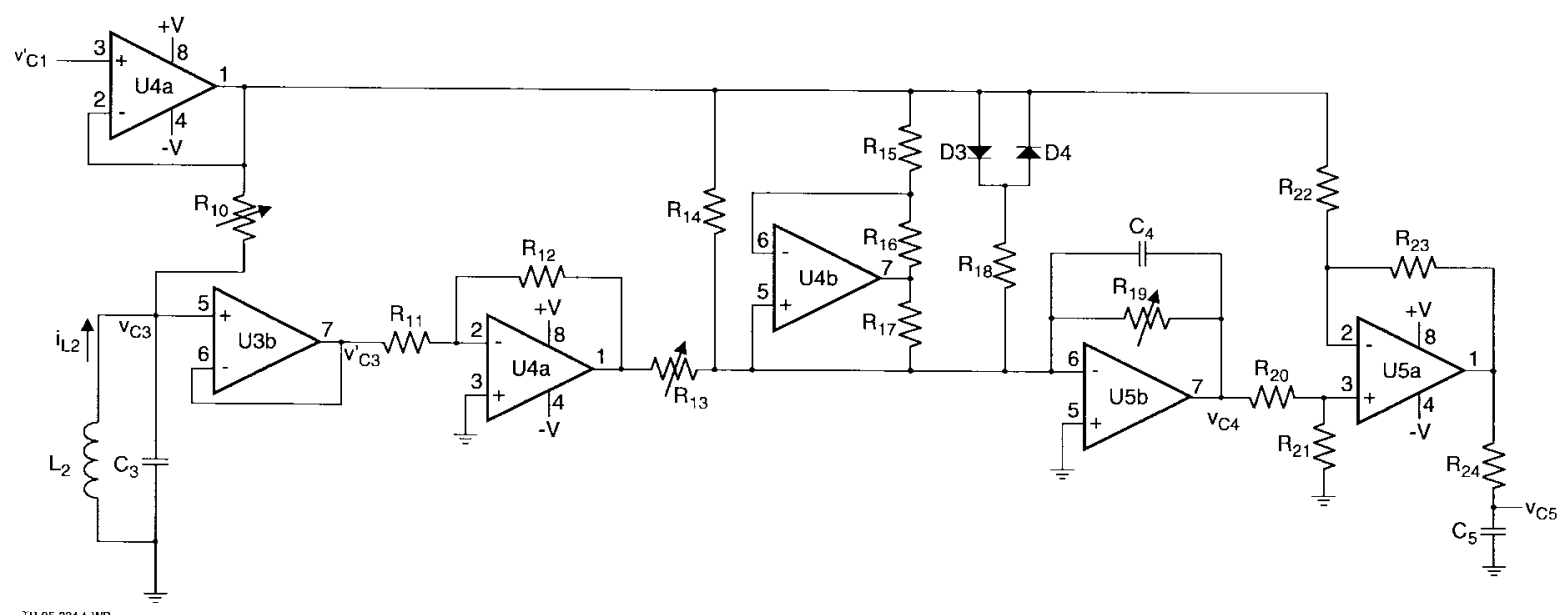

Fig. 5. Schematic for a receiver incorporating the nonlinear demodulation filter matched to the transmitter in shown in Fig. 4.

TABLE I

Circuit Component Values and Devices Used to Demonstrate Communications with Chaos

\begin{tabular}{l|l}
\hline \multicolumn{1}{c|}{ Component } & \multicolumn{1}{c}{ Value or Device } \\
\hline \hline $\mathrm{L}_{1}, \mathrm{~L}_{2}$ & $1.8 \mathrm{mH}$ \\
\hline $\mathrm{C}_{1}, \mathrm{C}_{4}$ & $0.001 \mu \mathrm{F}$ \\
\hline $\mathrm{C}_{2}, \mathrm{C}_{3}$ & $0.01 \mu \mathrm{F}$ \\
\hline $\mathrm{C}_{5}$ & $0.1 \mu \mathrm{F}$ \\
\hline $\mathrm{R}_{1}, \mathrm{R}_{2}, \mathrm{R}_{20}, \mathrm{R}_{21}$ & $10 \mathrm{k} \Omega$ \\
\hline $\mathrm{R}_{3}, \mathrm{R}_{4}, \mathrm{R}_{11}, \mathrm{R}_{12}, \mathrm{R}_{14}, \mathrm{R}_{22}, \mathrm{R}_{23}$ & $12 \mathrm{k} \Omega$ \\
\hline $\mathrm{R}_{5}, \mathrm{R}_{10}, \mathrm{R}_{13}, \mathrm{R}_{19}$ & $5-\mathrm{k} \Omega$ potentiometer \\
\hline $\mathrm{R}_{6}, \mathrm{R}_{7}, \mathrm{R}_{16}, \mathrm{R}_{17}$ & $220 \Omega$ \\
\hline $\mathrm{R}_{8}, \mathrm{R}_{15}$ & $750 \Omega$ \\
\hline $\mathrm{R}_{9}, \mathrm{R}_{18}$ & $1.2 \mathrm{k} \Omega$ \\
\hline $\mathrm{R}_{24}$ & $3.3 \mathrm{k} \Omega$ \\
\hline \hline $\mathrm{U} 1, \mathrm{U} 2, \mathrm{U} 3, \mathrm{U} 4, \mathrm{U} 5$ & $\mathrm{TL} 082$, Dual BiFET Op Amp \\
\hline $\mathrm{D} 1, \mathrm{D} 2, \mathrm{D} 3, \mathrm{D} 4$ & $1 \mathrm{~N} 914$, Silicon Diode \\
\hline
\end{tabular}

In order to match the receiver to the transmitter, the following design constraints are imposed:

$$
\begin{aligned}
L_{2} & =L_{1} \\
C_{3} & =C_{2} \\
C_{4} & =C_{1} \\
R_{10} & =R_{13}=R_{19}=R_{5} \\
R_{11} & =R_{12} \\
R_{14} & =R_{4} \\
R_{15} & =R_{8} \\
R_{16} & =R_{7} \\
R_{17} & =R_{6} \\
R_{18} & =R_{9} \\
R_{20} & =R_{21} \\
R_{22} & =R_{23} .
\end{aligned}
$$

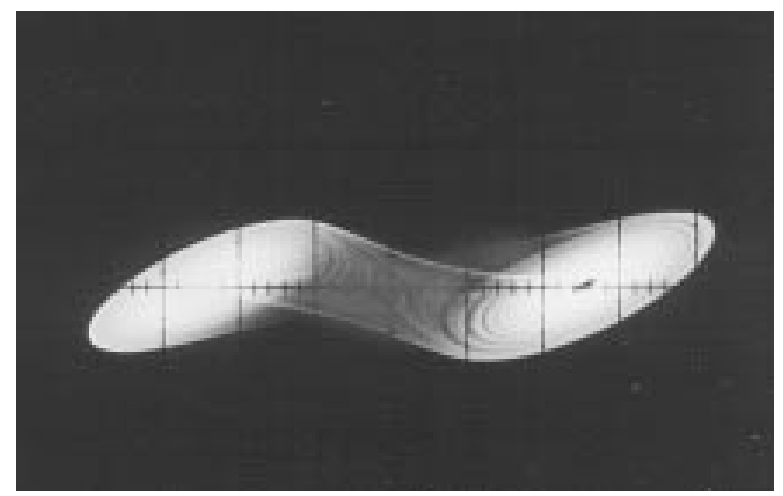

Fig. 6. Oscilloscope trace of the unmodulated attractor generated using the circuit shown in Fig. 4. (Vertical scale: $v_{C 2}^{\prime}, 0.5$ V/division; horizontal scale: $v_{C 1}^{\prime}, 0.5 \mathrm{~V} /$ division).

These constraints are sufficient conditions to assure tuning the receiver characteristics to those of the transmitter. However, these are not necessary conditions, as other configurations are also possible.

In practice, $R_{5}$ is adjusted to obtain a suitable chaotic carrier waveform; therefore, $R_{5}$ is implemented in the circuit using a potentiometer. As such, similar potentiometers are used for $R_{10}, R_{13}$, and $R_{19}$. These three potentiometers allow tuning the receiver for optimal output audio quality and provide a capability to compensate, to some extent, for imprecision in other matched circuit components. Convenient resistor values are chosen for the matched pairs $R_{11}=R_{12}, R_{20}=R_{21}$, and $R_{22}=R_{23}$, and the time constant $R_{24} C_{5}$ is chosen to set the filter parameter $q_{f}$.

Table I summarizes actual circuit values used for implementing the communications system. All component values in this table are listed as nominals, and all resistors are rated at $5 \%$ tolerance. The particular op amps and diodes indicated in this table are not critical, and comparable devices can be substituted.

For these component values, the circuit was adjusted to yield a suitable chaotic carrier with $R_{5}=1.315 \mathrm{k} \Omega$. A projection of the unmodulated attractor is shown in Fig. 6. This figure 


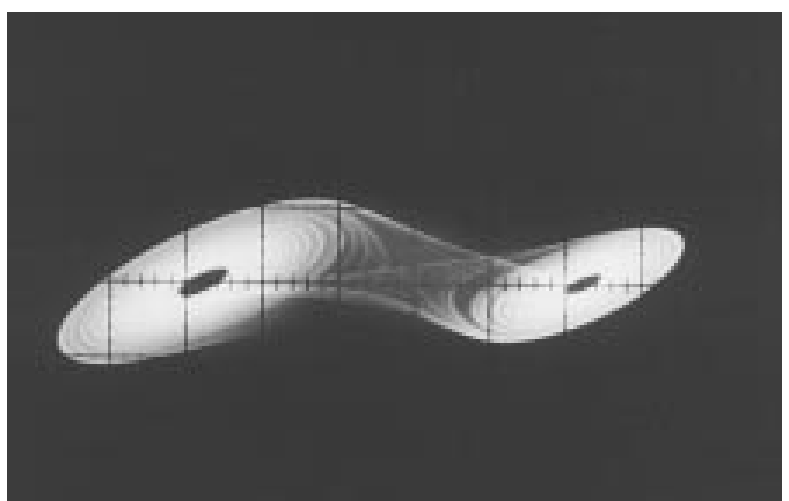

(a)

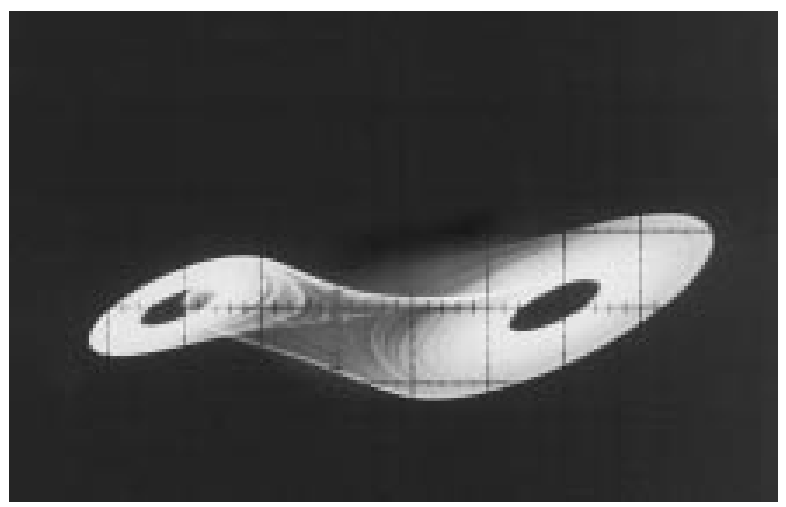

(b)

Fig. 7. Oscilloscope trace of the attractor generated using the circuit shown in Fig. 4 with an input modulation of (a) $V_{R}=+0.5 \mathrm{~V}\left(V_{L}=0 \mathrm{~V}\right)$ and (b) $V_{R}=-0.5 \mathrm{~V}\left(V_{L}=0 \mathrm{~V}\right)$. (Vertical scale: $v_{C 2}^{\prime}, 0.5 \mathrm{~V} /$ division; horizontal scale: $v_{C 1}^{\prime}, 0.5 \mathrm{~V} /$ division).

shows the familiar double-scroll attractor, which is identified by its dual-lobe structure.

The modulated attractor is shown in Fig. 7. In (a), a modulation of $v_{R}=+0.5 \mathrm{~V}\left(v_{L}=0 \mathrm{~V}\right)$ is applied, and it is seen that the attractor shifts to emphasize the left lobe. Conversely, in (b), a modulation of $v_{R}=-0.5 \mathrm{~V}\left(v_{L}=0 \mathrm{~V}\right)$ shifts the attractor to the right lobe. Based on these results, an ac signal would result in alternately emphasizing each of the lobes.

By adjusting the potentiometers $R_{10}, R_{13}$, and $R_{19}$, the receiver circuit was tuned for optimal audio output. For this tuning, a variety of audio signals were used, and optimality was judged on balancing signal clarity and reduced noise contamination. For this particular implementation, the best tuning was achieved with $R_{10}=1.332 \mathrm{k} \Omega, R_{13}=1.526$ $\mathrm{k} \Omega$, and $R_{19}=1.361 \mathrm{k} \Omega$, although these precise values are dependent on other circuit components that have not been fully characterized. In this tuning, the output audio quality is most sensitive to $R_{19}$. For this implementation, the output sound quality approaches that of standard AM radio transmissions. Certainly, voice signals are clear and easily understood.

Synchronization is achieved by adjusting $R_{10}$. Theoretically, $R_{10}$ should match $R_{5}$; however, imprecision in other "matched" circuit components results in a slight deviation of these two resistors. Evidence of the receiver synchronization is shown in Fig. 8, in which $v_{C 3}$ is strongly correlated with $v_{C 2}$, implying $y_{r}$ is synchronized with $y$.

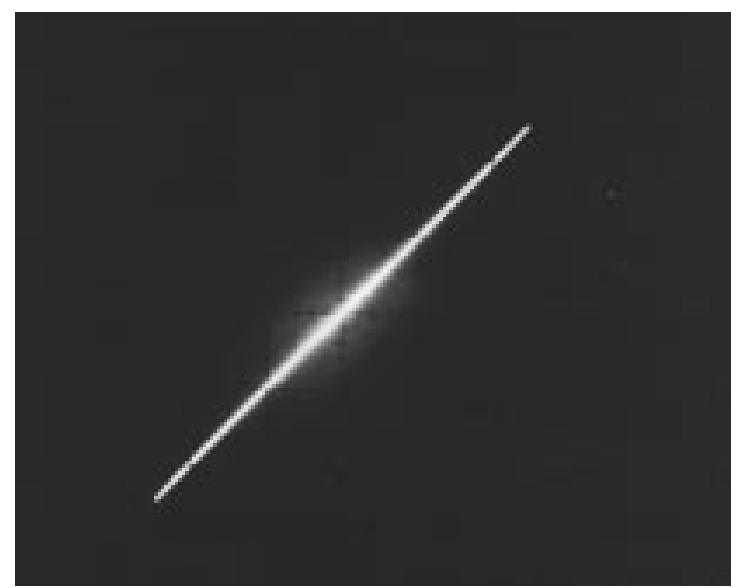

Fig. 8. Oscilloscope trace showing synchronization achieved in the first stage of the receiver shown in Fig. 5. (Vertical scale: $v_{C 3}^{\prime}, 0.2 \mathrm{~V} /$ division; horizontal scale: $v_{C 2}^{\prime}, 0.2 \mathrm{~V} /$ division).

The input and output traces shown in Fig. 9 demonstrate the effectiveness of the chaotic communication system for three different inputs. Specifically, the three input waveforms are (a) sinusoid, (b) triangle wave, and (c) square wave, each 500 $\mathrm{Hz}$ and 2-V peak-to-peak amplitude. In each of these, the top trace shows the input modulation at $v_{R}\left(v_{L}=0\right)$, while the bottom trace shows the system output at $v_{C 5}$. It is noted that some high-frequency content of the input is lost in this system, and this loss is most noticeable in the triangle- and squarewave samples. This filtering can be minimized by adjusting the filter constant $q_{f}$.

One of the important benefits of this communications architecture is that synchronization is maintained in the receiver even in the presence of modulation. Fig. 10 shows the synchronization that is achieved in the presence of the modulation used in Fig. 9(a). Specifically, the theory predicts that the receiver does not go out of tune as the transmitter is modulated. This follows from selecting a modulation parameter that does not appear in the synchronous subsystem. This design results in a consistent signal quality even for moderately large modulation signals.

\section{CONCLUSions}

In this paper, we described an innovative communications architecture using chaotic waveforms. Central to this architecture is a design for a nonlinear filter that recovers the information signal injected at the transmitter using parameter modulation. Using computer simulations, we showed the effectiveness and general applicability of this design. With an electrical circuit implementation, we demonstrated the practicality of our approach by transmitting and clearly receiving audio signals. These results imply that the design is tolerant to system perturbations and typical component mismatch between the transmitter and receiver circuits.

The system design possesses an interesting attribute that we have not explicitly illustrated in the simulations or hardware presented in this paper; namely, the dynamics of the chaos do not have to be spectrally separated from the information signal for the nonlinear filter to work. Specifically, an assumption in 


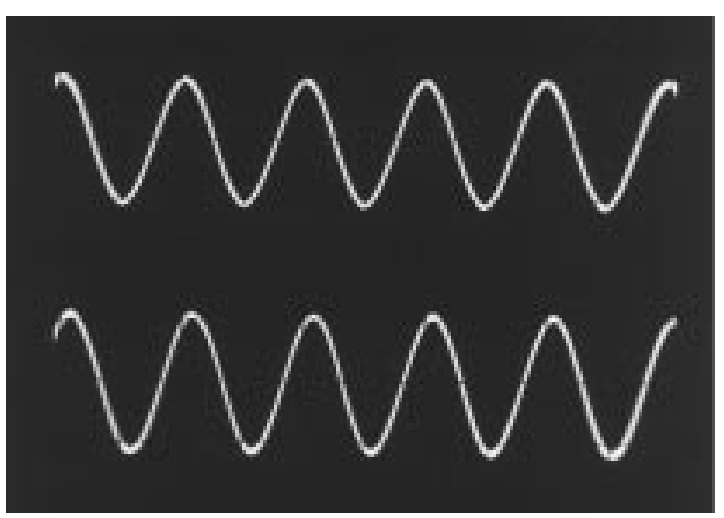

(a)

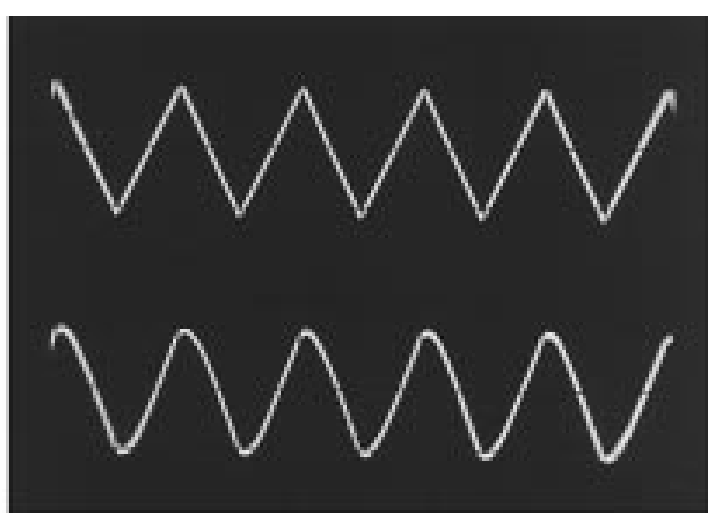

(b)

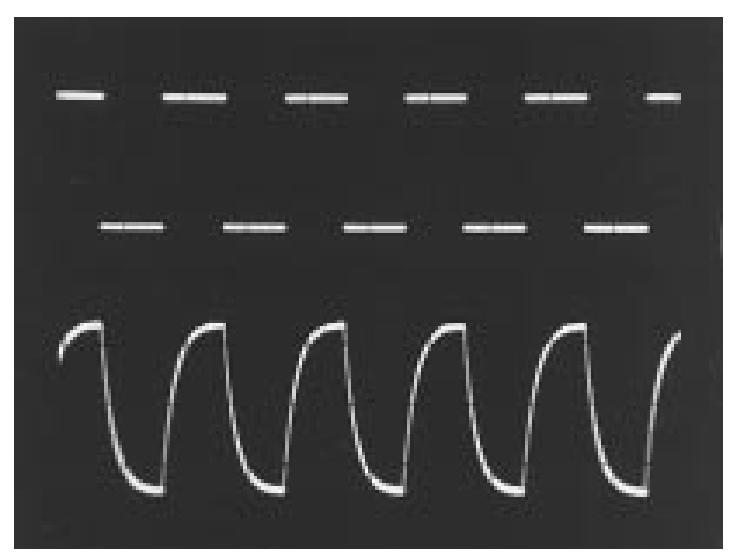

(c)

Fig. 9. Oscilloscope traces of input (top) and output (bottom) signals communicated using the transmitter shown in Fig. 4 and the receiver shown in Fig. 5 for (a) sine-, (b) triangle-, and (c) square-wave modulation. (Top vertical scale: $v_{R}, 1$ V/division; bottom vertical scale: $v_{C 5}$, 0.1 V/division; horizontal scale: $t, 1 \mathrm{~ms} /$ division).

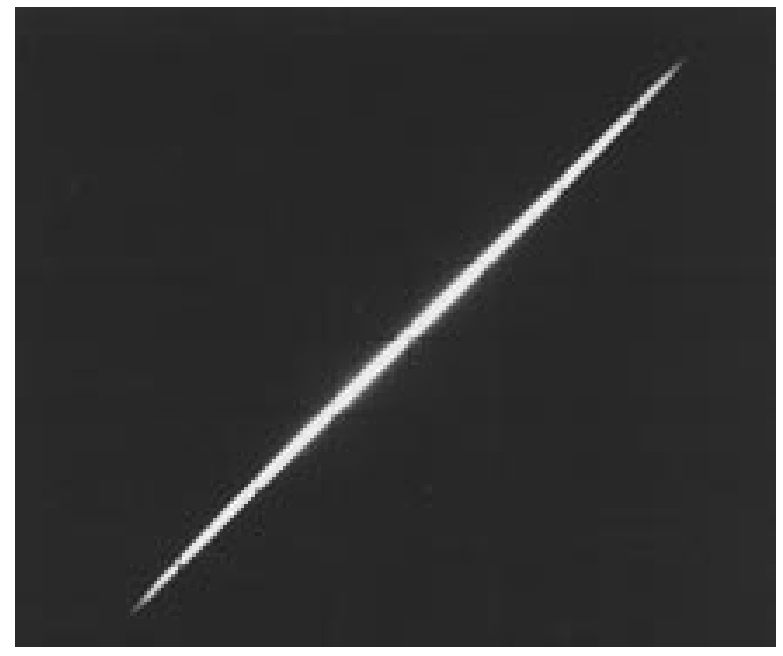

Fig. 10. Oscilloscope trace showing receiver synchronization that is maintained in the presence of modulation introduced at the transmitter. (Vertical scale: $v_{C 3}^{\prime}, 0.2 \mathrm{~V} /$ division; horizontal scale: $v_{C 2}^{\prime}, 0.2 \mathrm{~V} /$ division).

the filter derivation is that the information signal is slowly varying with respect to the time constant contained in the first filter constant, $k$; however, this requirement does not restrict the spectral content of the chaos. Therefore, with the proper choice of $k$, the dynamics of the information signal and the chaos can overlap significantly, thereby increasing the security aspects of the communications system. In light of this observation, we have exploited this attribute by developing an audio scrambling device. Although the analysis of Short [16] suggests high levels of security cannot be expected, we anticipate a chaotic scrambler based on the approach developed in this paper can provide a low level of security (or privacy) with only a minimal increase in bandwidth requirements. Our findings in developing this device will be documented in a future paper.

In view of applications for this technology, we are concerned with the impact that channel effects will impart on this communication system. Specifically, amplitude attenuation, bandwidth limitation, phase distortion, and channel noise are effects that may be encountered in fielded systems. Indeed, recent literature documents some practical investigations and initial design techniques that address these concerns [29], [31]-[34]. The characterization and mitigation of these effects are areas of interest in our current research and development efforts.

\section{REFERENCES}

[1] L. M. Pecora and T. L. Carroll, "Synchronization in chaotic systems," Phys. Rev. Lett., vol. 64, pp. 821-824, Feb. 1990.

[2] T. L. Carroll and L. M. Pecora, "Synchronizing chaotic circuits," IEEE Trans. Circuits Syst., vol. 38, pp. 453-456, Apr. 1991.

[3] _ "Synchronizing nonautonomous chaotic circuits," IEEE Trans. Circuits Syst. II, vol. 40, pp. 646-650, Oct. 1993. 
[4] M. J. Ogorzalek, "Taming chaos—Part I: Synchronization," IEEE Trans. Circuits Syst. I, vol. 40, pp. 693-699, Oct. 1993.

[5] J. M. T. Thompson and H. B. Stewart, Nonlinear Dynamics and Chaos. New York: Wiley, 1986.

[6] L. M. Pecora, "Overview of chaos and communications research," SPIE Proc., Chaos Commun., vol. 2038, pp. 2-25, July 1993.

[7] _ Ed., SPIE Proc., Chaos in Commun., vol. 2038, July 1993.

[8] M. Hasler, "Synchronization principles and applications," in Circuits and Systems Tutorials, C. Toumazou, Ed. Piscataway, NJ: IEEE, 1994, pp. 314-327.

[9] 1995 Int. Symp. Circuits Syst. (ISCAS'95), vols. I-III. Piscataway, NJ: IEEE, Apr. 1995

[10] J. M. Elmirghani, Ed., SPIE Proc., Chaotic Circuits Commun., vol. 2612, Oct. 1995.

[11] 1996 Int. Symp. Circuits Syst. (ISCAS'96), vol. 3. Piscataway, NJ: IEEE, May 1996.

[12] H. Dedieu, M. P. Kennedy, and M. Hassler, "Chaos shift keying: modulation and demodulation of a chaotic carrier using self-synchronizing Chua's circuits," IEEE Trans. Circuits Syst. II, vol. 40, pp. 634-642, Oct. 1993.

[13] A. V. Oppenheim, G. W. Wornell, S. H. Isabelle, and K. M. Cuomo, "Signal processing in the context of chaotic signals," in Proc. IEEE ICASSP, vol. IV, Mar. 1992, pp. 117-120.

[14] Lj. Kocarev, K. S. Halle, and K. Eckert, L. O. Chua, and U. Parlitz, "Experimental demonstration of secure communications via chaotic synchronization," Int. J. Bifurc. Chaos, vol. 2, no. 3, pp. 709-713, 1992.

[15] K. M. Cuomo, A. V. Oppenheim, and S. H. Strogatz, "Synchronization of Lorenz-based chaotic circuits with applications to communications," IEEE Trans. Circuits Syst. II, vol. 40, pp. 626-633, Oct. 1993.

[16] K. M. Short, "Steps for unmasking secure communications," Int. J. Bifurc. Chaos, vol. 4, no. 4, pp. 959-977, 1994.

[17] U. Parlitz, L. O. Chua, Lj. Kocarev, K. S. Halle, and A. Shang, "Transmission of digital signals by chaotic synchronization," Int. J. Bifurc. Chaos, vol. 2, no. 4, pp. 973-977, 1992.

[18] P. Celka, "Chaotic synchronization and modulation of nonlinear timedelayed feedback optical systems," IEEE Trans. Circuits Syst. I, vol. 42, pp. 455-463, Aug. 1995

[19] T. L. Carroll and L. M. Pecora, "Cascading synchronized chaotic systems," Phys. D, vol. 67, pp. 126-140, Aug. 1993.

[20] K. S. Halle, C. W. Wu, M. Itoh, and L. O. Chua, "Spread spectrum communication through modulation of chaos," Int. J. Bifurc. Chaos, vol. 3, no. 2, pp. 469-477, 1993.

[21] C. W. Wu and L. O. Chua, "A simple way to synchronize chaotic systems with applications to secure communications systems," Int. J. Bifurc. Chaos, vol. 3, no. 6, pp. 1619-1627, 1993.

[22] M. Itoh, H. Murakami, and L. O. Chua, "Communication systems via chaotic modulations," IEICE Trans. Fundament., vol. E77-A, no. 6, pp. 1000-1005, June 1994

[23] M. Itoh and H. Murakami, "New communication systems via chaotic synchronizations and modulations," IEICE Trans. Fundament., vol. E78A, no. 3, pp. 285-290, Mar. 1995.

[24] U. Feldmann, M. Hasler, and W. Schwarz, "Communication by chaotic signals: The inverse system approach," in Proc. IEEE ISCAS, vol. I, Apr. 1995, pp. 680-683.

[25] A. S. Dmitriev, A. I. Panas, and S. O. Starkov, "Experiments on speech and music signals transmission using chaos," Int. J. Bifurc. Chaos, vol. 5, no. 4, pp. 1249-1254, 1995.

[26] H. D. I. Abarbanel and P. S. Linsay, "Secure communications and unstable periodic orbits of strange attractors," IEEE Trans. Circuits Syst. II, vol. 40, pp. 643-645, Oct. 1993.
[27] D. R. Frey, "Chaotic digital encoding: an approach to secure communication," IEEE Trans. Circuits Syst. II, vol. 40, pp. 660-666, Oct. 1993.

[28] S. Hayes, C. Grebogi, and E. Ott, "Communicating with chaos," Phys. Rev. Lett., vol. 70, no. 20, pp. 3031-3034, May 17, 1993.

[29] P. A. Bernhardt, "Chaotic frequency modulation," SPIE Proc., Chaos Commun., vol. 2038, pp. 162-181, July 1993.

[30] M. P. Kennedy, "Three steps to chaos-Part II: A Chua's circuit primer," IEEE Trans. Circuits Syst. I, vol. 40, pp. 657-674, Oct. 1993.

[31] U. Parlitz and S. Ergezinger, "Robust communication based on chaotic spreading sequences," Phys. Lett. A, vol. 188, pp. 146-150, May 16, 1994.

[32] J. Schweizer and G. Setti, "Adaptive channel compensation for synchronization for chaotic Lure systems," in Proc. NDES'95, 1995, pp. 245-248.

[33] T. L. Carroll, "Amplitude-independent chaotic synchronization," Phys. Rev. E, vol. 53, no. 4, pp. 3117-3122, Apr. 1996.

[34] T. L. Carroll and L. M. Pecora, "The effect of filtering on communication using synchronized chaotic circuits," in Proc. IEEE ISCAS, vol. 3, May 1996, pp. 174-177.

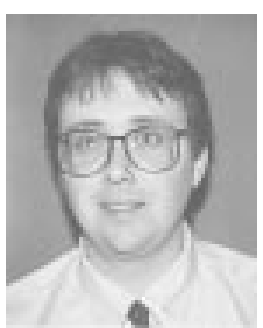

Ned J. Corron (M'93) was born in Geneva, IL, in 1962. He received the B.S. degree in mathematics and physics from North Central College in 1983 and the Ph.D. degree in applied mathematics from Northwestern University in 1989.

From 1984 to 1987 he was with the Physical Technologies Department of Amoco Corporation, Amoco Research Center, Naperville, IL. In 1990, he joined Dynetics, Inc., Huntsville, AL, where he is currently a staff scientist in the Sensor Systems and Technology Department. Presently, his research interests include wave propagation and scattering, nonlinear dynamics, and applications of chaos.

Dr. Corron is a member of the Society for Industrial and Applied Mathematics.

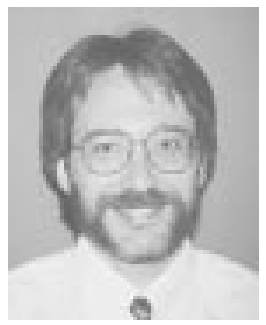

Daniel W. Hahs was born in Bonne Terre, MO, in 1956. He received the B.S. degree in electrical engineering from the University of Tennessee, Knoxville, in 1979 and the Ph.D. degree in physiology and biophysics from the University of Tennessee, Memphis, in 1986. His doctoral dissertation was in the area of electromyographic signal processing. He also received the M.S.E. degree from the University of Alabama, Huntsville, in 1988 specializing in control theory.

During 1979-1982 he was with Oak Ridge National Laboratory and Boeing Military Aircraft Company. From 1988 to the present he has been with Dynetics, Inc., Huntsville, AL. His current professional interests are in the areas of estimation, tracking, and data fusion. 